\title{
Effects of Ginsenosides, Active Ingredients of Panax ginseng, on Development, Growth, and Life Span of Caenorhabditis elegans
}

\author{
Joon-Hee Lee, ${ }^{a, \#}$ Sun-Hye Choi,,${ }^{b, \#}$ Oh-Seung Kwon, ${ }^{c}$ Tae-Joon ShIn, ${ }^{b}$ Jun-Ho LeE, ${ }^{b}$ \\ Byung-Hwan LeE, ${ }^{b}$ In-Soo Yoon, ${ }^{b}$ Mi Kyung Pyo, ${ }^{b}$ Hyewhon Rhim, ${ }^{c}$ Yoong-Ho Lim, ${ }^{d}$ \\ Yhong-Hee Shiм, ${ }^{d}$ Ji-yun AhN, ${ }^{e}$ Hyoung-Choon Kiм, ${ }^{f}$ David Joseph Chiтwood, ${ }^{g}$ \\ Sang-Mok LeE, ${ }^{*, b}$ and Seung-Yeol NAH ${ }^{*, b}$ \\ ${ }^{a}$ Department of Physical Therapy, Daebul University; 526-702, Korea: ${ }^{b}$ Ginsentology Research Laboratory and \\ Department of Physiology, College of Veterinary Medicine; 143-701, Korea: ${ }^{c}$ Bioanalysis/Biotransformation Research \\ Center and Life Science Division, KIST; Seoul 136-791, Korea: ${ }^{d}$ Bio/Molecular Informatics Center, Konkuk University; \\ Seoul 143-701, Korea: ${ }^{e}$ Food Function Research Division, Korea Food Research Institute; 483-746, Korea: \\ ${ }^{f}$ Neurotoxicology Program, College of Pharmacy, Kangwon National University; Chunchon 200-701, Korea: and \\ ${ }^{g}$ Nematology Laboratory, Department of Agriculture, Agricultural Research Service; Beltsville, MD 20705, U.S.A. \\ Received July 12, 2007; accepted August 16, 2007
}

The backbone structure of ginsenosides, active ingredients of Panax ginseng, is similar with that of sterol, especially cholesterol. Caenorhabditis elegans (C. elegans) is one of free living nematodes and is well-established animal model for biochemical and genetic studies. $C$. elegans cannot synthesize de novo cholesterol, although cholesterol is essential requirement for its growth and development. In the present study, we investigated the effects of ginseng total saponins (GTS) on the average brood size, growth, development, worm size, and life span of $C$. elegans in cholesterol-deprived and -fed medium. Cholesterol deprivation caused damages on normal growth, reproduction, and life span of worms throughout F1 to F3 generations. GTS supplement to cholesterol-deprived medium restored the growth, reproduction, and life span of worms as much as cholesterol alone-fed medium. GTS co-supplement to cholesterol-fed medium not only promoted worm reproduction but also induced bigger worms and faster growth than cholesterol-fed medium. In study to identify which ginsenosides are responsible for life span restoring effects of GTS, we found that ginsenoside Re supplement not only restored life span of worms grown in cholesterol-deprived medium but also prolonged life span of worms grown in cholesterol-fed medium. Worms grown in medium supplemented with ginsenoside $R_{b_{1}}$ or $R c$ to cholesterol-deprived medium exhibited strong filipin staining, in which filipin forms tight and specific complexes with $3 \beta$-hydroxy sterols. These results show a possibility that ginsenosides could be utilized by $C$. elegans as a sterol substitute and further indicate that ginsenoside $\mathrm{Rc}$ is the component of Panax ginseng that prolongs the life span of $C$. elegans.

Key words Panax ginseng; sterol; Caenorhabditis elegans; reproduction; life span; ginsenoside Rc

Ginseng, the root of Panax ginseng C. A. MEYER, has been used as a representative tonic for two thousand years in the Far East countries like Korea, China, and Japan. Now, ginseng is one of the most famous and precious herbal medicines consumed around the world. ${ }^{1)}$ Although ginseng exhibits multiple pharmacological actions in vitro or in vivo studies, its mechanisms on various efficacies are still elusive. Recent accumulating evidences show that ginseng saponins (or ginsenosides) are the main active ingredients of ginseng (Fig. 1). Ginseng root contains $3-4 \%$ of ginseng saponins. Ginseng saponins are especially abundant in fine roots rather than main body of ginseng root. Ginseng saponins are one of glycoside saponins and one of the derivatives of triterpenoid dammarane consisting of thirty carbon atoms. Each ginsenoside has a common hydrophobic four ring cholesterol-like backbone structure with sugar moieties attached. About 30 different types of ginseng saponins have been isolated and identified from the root of Panax ginseng (Fig. 1).

Caenorhabditis elegans ( $C$. elegans) is one of nematode species and is one of free living animals under the earth rather than is parasitic in host animals or plants. C. elegans takes its food from dead animals or plants. Since $C$. elegans has short life cycle and large amounts of worms can be easily grown for biochemical assays or genetic studies in the labo- ratory, the organism is one of the well-established genetic models and its development processes have well characterized. Interestingly, $C$. elegans cannot synthesize sterols unlike other
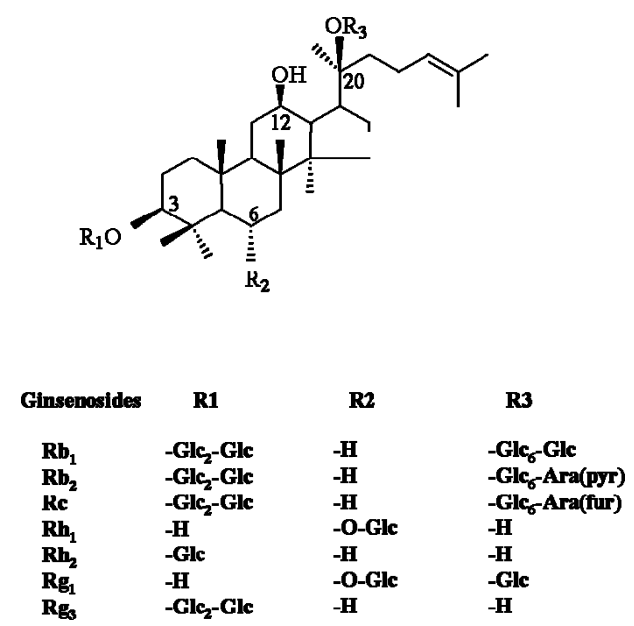

Fig. 1. Structures of the Representative Ginsenosides

They differ at three side chains attached the common steroid ring. Abbreviations for carbohydrates are as follows: Glc, glucopyranoside; Ara(pyr), arabinopyranoside; Rha, rhamnopyranoside. Superscripts indicate the carbon in the glucose ring that links the two carbohydrates.

* To whom correspondence should be addressed. e-mail: synah@konkuk.ac.kr; smlee@konkuk.ac.kr

\# These authors contributed equally to this work. 
animals and requires dietary sterol, which is usually supplied as cholesterol in the laboratory. ${ }^{2,3)}$ Although the functions of sterols in $C$. elegans have not been fully characterized, in vitro sterol deprivation results in decreased fertility, delayed development, and short life span throughout the first (F1), second (F2), and third (F3) generations., ${ }^{3,4)}$

On the other hand, some species of parasitic nematodes are found in wild and cultivated ginseng root. For example, Pratylenchus subpenetran is one of main parasitic animals and causes damage to ginseng roots. ${ }^{5)}$ It penetrates into fine roots or outer spaces of ginseng root from soil and makes a lot of small humps in fine roots, because they die if they are exposed to air. Its parasitic actions in fine roots of ginseng interfere to absorb nutrients from soil. It is known that if it is not treated with anti-nematode agents, it causes to severe damages on normal development of ginseng during young period, resulting in delay of the growth and further make ginseng fragile to other microbial infections. Although these observations suggest a possibility that ginseng roots might provide suitable environment(s) for survival of the parasitic nematodes, little is known on effects of ginseng saponins or individual ginsenosides as main ingredients of ginseng on the growth, development, and life span of nematodes.

We performed the present study with two-fold purposes: One was to examine whether supplements of ginseng total saponins (GTS) show any effects on the growth, development, and life span of $C$. elegans grown in cholesterol-deprived and -fed medium. If ginseng saponins exhibit physiological effects such as life span prolongation on $C$. elegans grown in cholesterol-deprived and -fed medium, the second aim was to identify which ginsenoside(s) are responsible for life span prolongation on C. elegans grown in cholesterol-deprived or -fed medium. We found that cholesterol deprivation induced significant damages on the average brood size, growth rate, percent development, and life span of $C$. elegans. However, GTS supplement to cholesterol-deprived medium restored the growth rate, percent development, and life span of worms as much as cholesterol-fed medium. We also found that GTS co-supplement to cholesterol-fed group not only increased the average brood size but also induced bigger worms and faster growth in perimeter from F1 to F3 generations compared with only cholesterol-fed group. We also found that ginsenoside $\mathrm{Rc}$ is responsible for restoration of life span of worms grown in cholesterol-deprived medium and further prolonged the life span of worms grown in cholesterol-fed medium. Ginsenoside $\mathrm{Rb}_{1}$ and $\mathrm{Rc}$ produced strong filipin staining of worms compared with cholesteroldeprived group. These results indicate that GTS, ginsenoside $\mathrm{Rb}_{1}$ or $\mathrm{Rc}$ supplement meets the sterol requirements of worms grown in sterol-deprived medium. Finally, the effects of GTS or ginsenosides to worms grown in cholesterol-deprived or -fed condition might be due to worms' ability to utilize the structural similarity of ginsenosides with sterol.

\section{MATERIALS AND METHODS}

Materials Electrophoresis grade agarose was obtained from Becton, Dickinson and Company (Sparks, MD, U.S.A.) and peptone was obtained from Amresco (Solon, $\mathrm{OH}$, U.S.A.). Cholesterol and all other analytical agents were obtained from Sigma (St. Louis, MO, U.S.A.). Cholesterol stock solution for cholesterol treatment group was prepared at $5 \mathrm{mg} / \mathrm{ml}$ of ethanol. The final ethanol concentration was $0.01 \%$. GTS and individual ginsenosides, isolated according to the method of Tanaka et al. (1966) and Shibata et al. (1966), respectively, was kindly provided by the Korea Ginseng Cooperation (Taejon, Korea). ${ }^{6,7)}$ Briefly, to remove phytosterols such as sitosterol or campasterol, the concentrated water extract was partitioned between water and $n$-hexane. The aqueous layer was further partitioned with butanol $(\mathrm{BuOH})$ to obtain $\mathrm{BuOH}$ fraction, ginseng total saponin fraction. The $\mathrm{BuOH}$ fraction, after concentration, was further processed to remove non-saponin components remaining in $\mathrm{BuOH}$ fraction using Diaion HP-20 column. ${ }^{8,9)}$ After chromatography, we confirmed no existence of phytosterols by HPLC. GTS contained ginsenoside $\mathrm{Rb}_{1} \quad(17.1 \%), \mathrm{Rb}_{2}$ (9.07\%), Rc (9.65\%), Rd (8.26\%), Re (9\%), Rf (3\%), $\operatorname{Rg}_{1}$ (6.4\%), $\operatorname{Rg}_{2}(4.2 \%), \operatorname{Rg}_{3}(3.8 \%), \operatorname{Ro}(3.8 \%), \operatorname{Ra}(2.91 \%)$ and other minor ginsenosides. GTS was diluted with nematode growth medium (NGM) before use. Figure 1 shows the structures of the eight representative ginsenosides.

Media and $\boldsymbol{C}$. elegans Growth The nematodes were grown and maintained on NGM agarose plates $(3 \mathrm{~g} / \mathrm{l} \mathrm{NaCl}$, $2.5 \mathrm{~g} / 1$ peptone, $5 \mathrm{mg} / \mathrm{l}$ cholesterol, $1 \mathrm{~mm} \mathrm{CaCl}, 1 \mathrm{mM} \mathrm{MgSO}_{4}$, $25 \mathrm{mM} \mathrm{KH}_{2} \mathrm{PO}_{4}$, pH 6.0, and $17 \mathrm{~g} / 1$ agar) with the Escherichia coli $\left(E\right.$. coli) OP50 strain in an incubator at $20^{\circ} \mathrm{C}$. ${ }^{10)}$ To obtain cholesterol-free conditions, agar was replaced by agarose, which was extracted three times with chloroform. ${ }^{11)}$ We also extracted peptone with ether in a large beaker in a fume hood. ${ }^{12)}$ For this, the peptone powder was mixed with an excess volume of ether, allowed to settle, decanted, and the process was repeated twice more. The extracted peptone was allowed to dry overnight in the hood to remove the remaining ether. ${ }^{12)}$ E. coli strain OP50 was also grown directly in this sterol-free medium. Wild-type N2 C. elegans (Bristol type) was provided by the Caenorhabditis Genetics Center and was maintained according to the methods of Brenner (1974). ${ }^{10)}$ For definition of various generations of worms, we first cultured worms (Po, $n=10-20)$ in each treatment group such as cholesterol-deprived, cholesterol ( $5 \mu \mathrm{g} / \mathrm{ml}$ )-fed group, various concentrations of GTS + cholesterol-deprived, or GTS + cholesterol $(5 \mu \mathrm{g} / \mathrm{ml})$-fed group. When eggs from Po animals grown in each treatment were again placed on various treatment group plates as mentioned above, the resulting animals are referred to as F1 generation. When eggs from F1 animals were grown in various treatment group plates, the resulting animals are referred to as F2 generation. Again when eggs from F2 animals were grown in various treatment group plates, the resulting animals are referred to as F3 generation.

Analyses of Brood Size and Development The effects of GTS on brood sizes and percent development were examined at $20^{\circ} \mathrm{C}$. Gravid adults $(n=10-20)$ were first allowed to lay eggs for $1 \mathrm{~h}$ in agarose plate of cholesterol-deprived, cholesterol-fed, various concentrations of GTS + cholesteroldeprived, or GTS+cholesterol-fed group and then were removed. The gravid adults (F1) $(n=10-20)$ grown from eggs of each treatment group were again allowed to lay eggs for $1 \mathrm{~h}$ and then were removed. The resulting synchronous population was raised until the late L4 stage. L4 stage worms were again individually cloned onto agarose plates with cholesterol-deprived, cholesterol-fed, various concentrations of 
GTS + cholesterol-deprived, or GTS + cholesterol-fed group. The individual worms were transferred to new plate every $8-16 \mathrm{~h}$ until the end of the reproductive period. The brood size is defined as the sum of non-hatched and hatched embryos. Percent development is defined as the number of hatched embryos that reach adulthood. ${ }^{13)}$ An average value was obtained from three independent experiments. We analyzed brood sizes and percent development of F2 and F3 progenies with the same procedure as described above using F1 and F2 progenies of each treatment group, respectively.

Measurement of Growth Rate and Worm Length L4 hermaphrodites $(n=20-30)$ grown in cholesterol-deprived, cholesterol-fed, various concentrations of GTS + cholesteroldeprived, or GTS + cholesterol-fed group were individually cloned onto agarose plates at $20^{\circ} \mathrm{C}$. Growth rate of worms on each agarose plate was determined by observation of characteristic stage-specific morphology every $24 \mathrm{~h}$ until the end of the growth. We put individual worm at a specific stage on an agarose pad and measured the length of it. We also analyzed growth rate of F2 and F3 progenies with the same procedure as described above using F1 and F2 progenies of each treatment group, respectively.

Measurement of Life Span For synchronous culture, $\mathrm{L} 1$ larvae were allowed to hatch by overnight at $20^{\circ} \mathrm{C}$ incubation in M9 buffer and transferred to different media plates to develop to L4 larvae. L4 larvae were transferred to NGM plates supplemented with $40 \mu \mathrm{M}$ 5-fluoro-2'-deoxyuridine (FuDR) to suppress the production of their progenies. NGM agarose-FuDR plates were also divided into cholesterol-deprived, cholesterol-fed, GTS or ginsenoside + cholesterol-deprived, or GTS or ginsenoside+cholesterol-fed group. The worms were examined daily and dead worms, which did not move after touching their heads with a platinum wire, were removed to count. The dead worms due to scrawling up the walls of plates were excluded from this analysis. The worms on the plates contaminated by other microorganisms were also excluded from analysis, since lifespan of worms were affected by the concentration of dietary bacteria throughout F1 to F3 generations. The life span was determined from the percentage of worms alive on a given day.

Filipin Staining Filipin staining was performed according to the methods of Merris et al. (2003). ${ }^{3)}$ Briefly, worms (30 mm dishes) grown with cholesterol-deprived, cholesterolfed, ginsenoside $\mathrm{Rb}_{1}$ or $\mathrm{Rc}+$ cholesterol-deprived group were washed into a $15 \mathrm{ml}$ conical centrifuge tube and washed three times with ice-cold distilled water. After aspiration of the last wash, the volume was adjusted to $200 \mu \mathrm{l}$, and an equal volume of ice-cold $2 \mathrm{X} \mathrm{RFB},{ }^{14)}$ plus $25 \mu \mathrm{l}$ of $37 \%$ formaldehyde solution, $2.5 \mu \mathrm{l}$ of $\beta$-mercaptoethanol, and $1 \mu \mathrm{l}$ of phenoxypropanol were added. The sample was freeze-thawed three times in an ethanol-dry ice bath and $25 \mu \mathrm{l}$ of freshly prepared filipin III $(10 \mathrm{mg} / \mathrm{ml}$ in DMSO $)$ was added. The sample was flushed with nitrogen, capped, and incubated for $1-4 \mathrm{~h}$ in an ice-water bath in the dark. It was then washed three times with PBS. After aspiration, $5 \mu \mathrm{l}$ of liquid from the bottom of the conical tube containing the highest concentration of animals, were transferred to a glass slide using a plastic pipette tip that had been cut with a razor to enlarge the diameter of the aperture to $1 \mathrm{~mm}$. Five microliters of Vectashield (Vector Laboratories, Inc.) was added, and the mixture was viewed under a $22 \times 22 \mathrm{~mm}$ cover slip. Maximum absorption (fluorescence excitation) of filipin is at $338 \mathrm{~nm}$, emission at $480 \mathrm{~nm}$. Filipin-stained animals were viewed using the same filter set used for DAPI staining.

\section{RESULTS}

Effects of GTS on Worm Reproduction and Growth Ability in Cholesterol-Deprived or -Fed Medium Worms grown in NGM agarose plates at $20^{\circ} \mathrm{C}$ were divided into four groups: cholesterol-deprived, cholesterol $(5 \mu \mathrm{g} / \mathrm{ml})-\mathrm{fed}, \mathrm{GTS}$ $(5,100$, or $300 \mu \mathrm{g} / \mathrm{ml})+$ cholesterol-deprived, and GTS (300 $\mu \mathrm{g} / \mathrm{ml})+$ cholesterol $(5 \mu \mathrm{g} / \mathrm{ml})$-fed group $(n=20-30$, each group). We first examined the effects of GTS on the average brood size of worms. The average brood size is defined as the sum of non-hatched and hatched embryos. An embryo was scored as being dead if it had not hatched after $24 \mathrm{~h}$ at $20^{\circ} \mathrm{C}$. The average brood size of worms grown in cholesterol-deprived, various concentrations of GTS + cholesterol-deprived, or GTS + cholesterol-fed medium in F1, F2 and F3 generations was summarized in Table 1 . We found that GTS supplement to cholesterol-deprived medium restored the average brood size of worms with concentration-dependent manners throughout $F 1$ to $F 3$ generations with full recovery of the brood size at GTS $(300 \mu \mathrm{g} / \mathrm{ml})$. Interestingly, co-supplement of GTS $(300 \mu \mathrm{g} / \mathrm{ml})$ to cholesterol-fed group more significantly increased the average brood size of worms compared with cholesterol alone-fed group throughout F1 to F3 generations (Table 1). These results indicate that cholesterol deprivation in medium exhibited the deleterious effects on normal C. elegans reproduction as previous reports, ${ }^{3,4}$ but that GTS supplement made a normal egg-laying even in cholesterol de-

Table 1. Effects of GTS on Average Brood Size and Development of $C$. elegans Grown in Cholesterol-Deprived and -Fed Medium

\begin{tabular}{|c|c|c|}
\hline F1 & Average brood size & Percent development \\
\hline- Chol & $111 \pm 26.7(39 \%)^{\# \#}$ & $64.0 \pm 7.1^{\# \#}$ \\
\hline- Chol+GTS $5(\mu \mathrm{g} / \mathrm{ml})$ & $217 \pm 13.2(76 \%)^{*, \# \#}$ & $95.8 \pm 2.4^{*}, \# \#$ \\
\hline+ GTS 100 & $216 \pm 12.7(76 \%)^{* * \text {,\#\# }}$ & $88.7 \pm 2.5^{*}$,\#\# \\
\hline+ GTS 300 & $292 \pm 10.1(102 \%)^{* *}$ & $99.7 \pm 0.3 *$ \\
\hline+ Chol & $286 \pm 7.7(100 \%)^{* *}$ & $98.8 \pm 0.6^{*}$ \\
\hline+ Chol+GTS 300 & $325 \pm 8.9(114 \%)^{* *, \#}$ & $99.7 \pm 0.2 *$ \\
\hline $\mathrm{F} 2$ & Average brood size & Percent development \\
\hline- Chol & $45 \pm 6.3(16 \%)^{\# \#}$ & $65.9 \pm 9.5^{\# \#}$ \\
\hline- Chol+GTS 5 & $144 \pm 25.3(50 \%)^{*, \# \#}$ & $90.5 \pm 3.6^{*, \# \#}$ \\
\hline+ GTS 100 & $232 \pm 17.1(81 \%)^{* *, \#}$ & $95.1 \pm 0.7^{*, \# \#}$ \\
\hline+ GTS 300 & $276 \pm 6.7(97 \%)^{* *}$ & $99.7 \pm 0.2 *$ \\
\hline+ Chol & $286 \pm 7.7(100 \%)^{* *}$ & $98.8 \pm 0.6^{*}$ \\
\hline+ Chol+GTS 300 & $307 \pm 7.0(107 \%)^{* *}$ & $99.2 \pm 0.4 *$ \\
\hline $\mathrm{F} 3$ & Average brood size & Percent development \\
\hline -Chol & $47 \pm 12.3(16 \%)^{\# \#}$ & $63.7 \pm 5.9^{\# \#}$ \\
\hline- Chol+GTS 5 & $137 \pm 10.0(48 \%)^{* *, \# \#}$ & $89.3 \pm 2.3 *$,\#\# \\
\hline+ GTS 100 & $242 \pm 15.2(85 \%)^{* *, \#}$ & $99.0 \pm 0.3^{*, \#}$ \\
\hline+ GTS 300 & $312 \pm 16.9(109 \%)^{* *}$ & $99.9 \pm 0.1 *$ \\
\hline+ Chol & $286 \pm 7.7(100 \%)^{* *}$ & $98.8 \pm 0.6^{*}$ \\
\hline+ Chol+GTS 300 & $334 \pm 8.3(117 \%)^{* *, \#}$ & $98.7 \pm 0.4^{*}$ \\
\hline
\end{tabular}

$* p<0.01, * * p<0.001$, significantly different from cholesterol-deprived group $\# p<0.01$, \# $p<0.001$, significantly different from cholesterol-fed group. The percentages in the parentheses in average brood size section are obtained from the percent ratio of $+\operatorname{chol}(5 \mu \mathrm{g} / \mathrm{ml})$ to other treatments. 
Table 2. Effects of GTS on Length and Growth Rate of C. elegans Grown in Cholesterol-Deprived and -Fed Medium

\begin{tabular}{|c|c|c|c|c|c|}
\hline \multirow{2}{*}{$\mathrm{F} 1$} & \multicolumn{4}{|c|}{ Worm length (mm) } & \multirow{2}{*}{$\begin{array}{c}\text { Growth rate } \\
\text { Egg to Adult (h) }\end{array}$} \\
\hline & L3 & L4 & Young adult & Adult & \\
\hline- Chol & $0.4 \pm 0.01$ & $0.8 \pm 0.01$ & $1.0 \pm 0.02$ & $1.1 \pm 0.02$ & $115 \pm 0.2$ \\
\hline$-\mathrm{Chol}+\mathrm{GTS} 5(\mu \mathrm{g} / \mathrm{ml})$ & $0.5 \pm 0.02 *$ & $0.9 \pm 0.01 *$ & $1.1 \pm 0.02 *$ & $1.2 \pm 0.03 *$ & $110 \pm 0.2 *$ \\
\hline+ GTS 100 & $0.5 \pm 0.02 *$ & $0.9 \pm 0.02 *$ & $1.2 \pm 0.01^{* *}$ & $1.2 \pm 0.03 *$ & $100 \pm 0.1^{* *}$ \\
\hline+ GTS 300 & $0.5 \pm 0.02 *$ & $1.0 \pm 0.03 * *$ & $1.2 \pm 0.01 * *$ & $1.3 \pm 0.03 * *$ & $94 \pm 0.1^{* *}$ \\
\hline+ Chol & $0.5 \pm 0.02 *$ & $1.0 \pm 0.02 * *$ & $1.2 \pm 0.02 * *$ & $1.4 \pm 0.03 * *,(40.5 \pm 0.9)$ & $96 \pm 0.1 * *$ \\
\hline+ Chol+GTS 300 & $0.5 \pm 0.02 *$ & $1.1 \pm 0.02 * *$ & $1.3 \pm 0.02 * *$ & $1.5 \pm 0.03^{* *},(53.1 \pm 0.4)^{\#}$ & $92 \pm 0.1 * *, \#$ \\
\hline \multirow{2}{*}{$\mathrm{F} 2$} & \multicolumn{4}{|c|}{ Worm length (mm) } & Growth rate \\
\hline & L3 & L4 & Young adult & Adult & Egg to Adult (h) \\
\hline- Chol & $0.3 \pm 0.01$ & $0.5 \pm 0.02$ & $0.8 \pm 0.04$ & $1.1 \pm 0.04$ & $123 \pm 0.3$ \\
\hline- Chol+GTS 5 & $0.4 \pm 0.01 *$ & $0.6 \pm 0.02 *$ & $1.0 \pm 0.03 * *$ & $1.3 \pm 0.02 * *$ & $115 \pm 0.2 *$ \\
\hline+ GTS 100 & $0.4 \pm 0.02 *$ & $0.7 \pm 0.03 * *$ & $1.1 \pm 0.01 * *$ & $1.3 \pm 0.02 * *$ & $102 \pm 0.1^{* *}$ \\
\hline+ GTS 300 & $0.4 \pm 0.01 *$ & $0.8 \pm 0.01 * *$ & $1.2 \pm 0.02 * *$ & $1.5 \pm 0.02 * *$ & $94 \pm 0.1 * *$ \\
\hline+ Chol & $0.4 \pm 0.01 *$ & $0.8 \pm 0.01 * *$ & $1.2 \pm 0.02 * *$ & $1.4 \pm 0.02 * *,(40.9 \pm 0.8)$ & $96 \pm 0.1 * *$ \\
\hline +Chol+GTS 300 & $0.4 \pm 0.01^{*}$ & $0.8 \pm 0.01 * *$ & $1.2 \pm 0.01 * *$ & $1.5 \pm 0.02 * *,(54.8 \pm 0.7)^{\#}$ & $92 \pm 0.1 * *, \#$ \\
\hline \multirow{2}{*}{ F3 } & \multicolumn{4}{|c|}{ Worm length (mm) } & Growth rate \\
\hline & L3 & L4 & Young adult & Adult & Egg to Adult (h) \\
\hline- Chol & $0.4 \pm 0.01$ & $0.6 \pm 0.01$ & $0.9 \pm 0.04$ & $1.0 \pm 0.03$ & $136 \pm 0.3$ \\
\hline -Chol+GTS 5 & $0.4 \pm 0.01$ & $0.7 \pm 0.01 *$ & $1.1 \pm 0.01^{*}$ & $1.3 \pm 0.01 * *$ & $126 \pm 0.2 *$ \\
\hline+ GTS 100 & $0.4 \pm 0.03$ & $0.8 \pm 0.02 * *$ & $1.2 \pm 0.01 * *$ & $1.4 \pm 0.02 * *$ & $104 \pm 0.1 * *$ \\
\hline+ GTS 300 & $0.5 \pm 0.01 *$ & $1.0 \pm 0.03 * *$ & $1.3 \pm 0.02 * *$ & $1.5 \pm 0.01 * *$ & $94 \pm 0.1 * *$ \\
\hline+ Chol & $0.5 \pm 0.01 *$ & $1.0 \pm 0.02 * *$ & $1.2 \pm 0.01 * *$ & $1.4 \pm 0.01 * *,(41.2 \pm 0.8)$ & $96 \pm 0.1 * *$ \\
\hline +Chol+GTS 300 & $0.5 \pm 0.01 *$ & $1.0 \pm 0.01 * *$ & $1.3 \pm 0.01 * *$ & $1.5 \pm 0.01 * *,(55.0 \pm 0.7)^{\#}$ & $92 \pm 0.1 * *, \#$ \\
\hline
\end{tabular}

$* p<0.01, * * p<0.001$, significantly different from cholesterol-deprived group. \# $p<0.01$, significantly different from cholesterol-fed group. The numbers of ( ) in adult stage of each generation indicate body diameter $(\mu \mathrm{m})$ of worms.

prived medium. Interestingly, co-supplement of GTS to cholesterol-fed group exhibited additional effects by further increasing the egg-laying ability of $C$. elegans (Table 1 ).

We next examined the percent development of worms from an embryo to adult in cholesterol-deprived, cholesterolfed, GTS + cholesterol-deprived, and GTS + cholesterol-fed medium as above. Percent development is defined as the number of hatched embryos that reach adulthood. ${ }^{13)}$ For this, we examined twenty to thirty cloned hermaphrodites. Percent development of worms grown in various conditions was summarized in Table 1. We found that GTS supplement to cholesterol-deprived medium also restored the percent development of worms with concentration-dependent manners throughout F1 to F3 generations with full recovery of the percent development at GTS $(300 \mu \mathrm{g} / \mathrm{ml})$. In contrast to the average brood size, co-supplement of GTS $(300 \mu \mathrm{g} / \mathrm{ml})$ to cholesterol-fed group did not affect worm development. These results indicate that GTS supplement could promote egg-laying ability and help normal developments of $C$. elegans to adulthood in cholesterol-deprived state.

Effects of GTS on Worm Length in Cholesterol-Deprived or -Fed Medium Next, we have investigated the effects of various concentrations of GTS on worm length grown in cholesterol-deprived or -fed medium. We measured the worm length from L3 to adult stages. For this, we have first synchronized worms and divided twenty worms into each experimental plate. After $24 \mathrm{~h}$ incubation in medium of each group, we have started to measure the worm length. The comparisons among various treatment groups in worm length from L3 to adult stage were summarized in Table 2. In F1, F2 and F3 generations, we could observe the significant differences in the worm length between worms grown in GTS supplement $(5,100$ or $300 \mu \mathrm{g} / \mathrm{ml})$ and worms grown in cholesterol-deprived medium at adult stage. However, only GTS supplement $(300 \mu \mathrm{g} / \mathrm{ml})$ to cholesterol-deprived medium recovered the worm length as much as cholesterol-fed group (Fig. 2, Table 2). Thus, GTS supplement to cholesteroldeprived medium increased worm length with concentrationdependent manners. Interestingly, at adult stages, the growth of worms in each group reached plateau state with prominent differences in worm length except GTS + cholesterol-fed groups. Moreover, co-supplement of GTS to cholesterol-fed medium produced significant bigger worms in size and diameter than those grown in cholesterol-fed medium (Fig. 2, Table 2). These results indicate that GTS restores worm growths in cholesterol-deprived medium to normal size with concentration-dependent manners and that co-supplement GTS to cholesterol-fed medium promotes worm growths.

Effects of GTS on Worm Growth Rate in CholesterolDeprived or -Fed Medium We next examined the effects of various concentrations of GTS on the growth rate in cholesterol-deprived or -fed medium. As shown in Table 2, the growth rates (i.e. the time to reach at adult stage from egg) of worms grown in cholesterol-deprived medium were significantly retarded by 19,27 , and $40 \mathrm{~h}$ at F1, F2, and F3 generations, respectively, compared with worms grown in choles- 


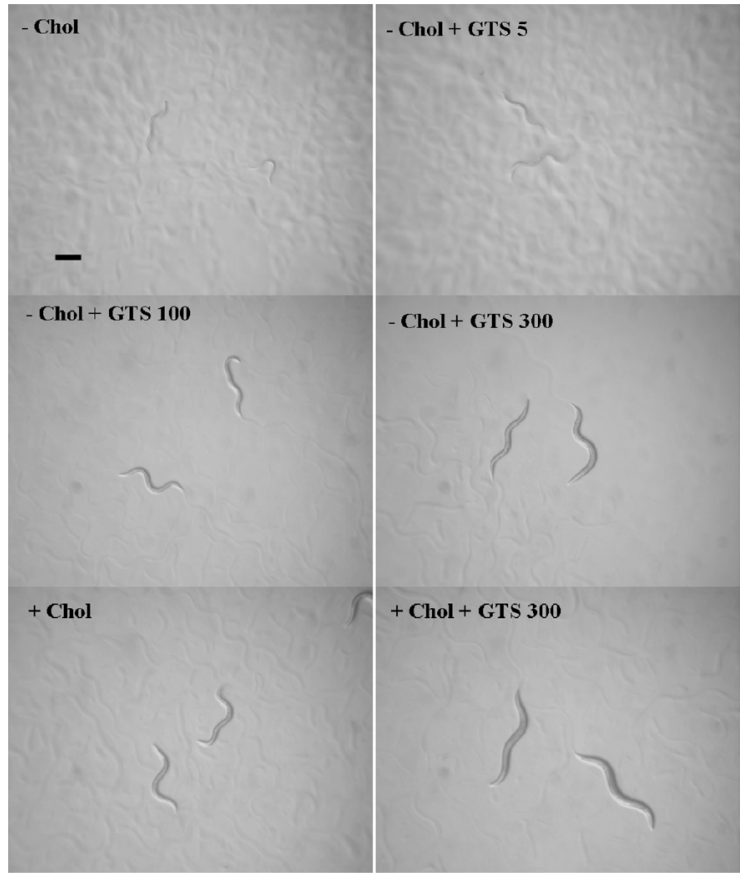

Fig. 2. Effects of GTS on Worm Size Grown in Cholesterol-Deprived or -Fed Medium

Eggs laid by F1 animals were placed on plates containing the following treatments: -Chol (cholesterol-deprivation); -Chol+GTS 5 (cholesterol deprivation $+5 \mu \mathrm{g} / \mathrm{ml}$ GTS); - Chol+GTS 100 (cholesterol-deprivation + $100 \mu \mathrm{g} / \mathrm{ml}$ GTS); - Chol+GTS 300 (cholesterol deprivation $+300 \mu \mathrm{g} / \mathrm{ml}$ GTS); +Chol (cholesterol-fed, $5 \mu \mathrm{g} / \mathrm{ml}$ ), or + Chol + GTS 300 (cholesterol-fed, $5 \mu \mathrm{g} / \mathrm{ml}+300 \mu \mathrm{g} / \mathrm{ml} \mathrm{GTS}$ ). Photographs were taken after growth for $72 \mathrm{~h}$ of $\mathrm{F} 2$ at $20^{\circ} \mathrm{C}$. Scale bar, $200 \mu \mathrm{m}$. All photographs were taken at the same time under various treatment conditions.

terol-fed medium. However, we could observe that GTS supplement to cholesterol-deprived medium restored the growth rate with concentration-dependent manners. Although GTS $(5,100 \mu \mathrm{g} / \mathrm{ml})+$ cholesterol-deprived medium significantly retarded the growth rate compared with cholesterol-fed group from F1 to F3 generations, GTS $(300 \mu \mathrm{g} / \mathrm{ml})+$ cholesterol-deprived medium produced almost the same growth rate as observed in cholesterol-fed medium from F1 to F3 generations. Interestingly, worms grown in GTS+cholesterol-fed medium exhibited significantly more faster growth than those grown in cholesterol alone-fed medium (Table 2). These results indicate that GTS supplement not only restored growth retardation of worms observed in cholesterol-deprived medium but also promoted rapid worm growth in cholesterol-fed medium.

Effects of GTS on Worm Life Span in Cholesterol-Deprived or -Fed Medium Since worms grown in cholesterol deprived-medium show shorter life span than those grown in cholesterol-fed medium, ${ }^{4,15)}$ we also examined whether GTS supplement to cholesterol-deprived medium affects worm life span. For this, L1 larvae were allowed to hatch by overnight at $20^{\circ} \mathrm{C}$ incubation in M9 buffer and transferred to different kinds of plates to develop to L4 larvae. L4 larvae were transferred to NGM plates supplemented with FuDR to suppress the production of their progenies. The synchronized L4 worms were again divided into cholesterol-deprived $(n=100)$, cholesterol (5 or $50 \mu \mathrm{g} / \mathrm{ml})$-fed $(n=118)$, GTS $(5$, 100 , or $300 \mu \mathrm{g} / \mathrm{ml})+$ cholesterol deprived $(n=95-100$ for each concentration), and cholesterol-fed+GTS $(300 \mu \mathrm{g} / \mathrm{ml})$ $(n=98)$ in the presence of FuDR. The average life span and
Table 3. Effects of GTS on Life Span of C. elegans Grown in CholesterolDeprived and -Fed Medium

\begin{tabular}{llc}
\hline \hline \multirow{2}{*}{ F1 } & \multicolumn{2}{c}{ Life span } \\
\cline { 2 - 3 } & Mean days & Maximum days \\
\hline - Chol & $12.8 \pm 0.2^{\text {\#\# }}$ & 15 \\
- Chol+GTS 5 $(\mu \mathrm{g} / \mathrm{ml})$ & $16.5 \pm 0.1^{* *}$ & 20 \\
+ GTS 100 & $17.2 \pm 0.4^{* *}$ & 22 \\
+ GTS 300 & $17.4 \pm 0.3^{* *}$ & 24 \\
+ Chol 5 & $16.5 \pm 0.2^{* *}$ & 20 \\
+ Chol + GTS 300 & $15.7 \pm 0.6^{*}$ & 21 \\
+ Chol 50 & $15.0 \pm 0.1^{*}$ & 19 \\
\hline
\end{tabular}

\begin{tabular}{ccc}
\hline \hline \multirow{2}{*}{ F2 } & \multicolumn{2}{c}{ Life span } \\
\cline { 2 - 3 } & Mean days & Maximum days \\
\hline - Chol & $13.7 \pm 0.4^{\#}$ & 17 \\
- Chol+GTS 5 & $16.2 \pm 0.0^{*}$ & 20 \\
+ GTS 100 & $17.5 \pm 0.3^{*}$ & 23 \\
+ GTS 300 & $16.4 \pm 0.1^{*}$ & 22 \\
+ Chol 5 & $16.2 \pm 0.2^{*}$ & 20 \\
+ Chol+GTS 300 & $16.7 \pm 0.1^{*}$ & 22 \\
+ Chol 50 & $16.5 \pm 0.1^{*}$ & 21 \\
\hline
\end{tabular}

\begin{tabular}{llc}
\hline \hline \multirow{2}{*}{ F3 } & \multicolumn{2}{c}{ Life span } \\
\cline { 2 - 3 } & Mean days & Maximum days \\
\hline - Chol & $13.4 \pm 0.3^{\# \#}$ & 17 \\
- Chol+GTS 5 & $17.7 \pm 0.2^{* *}$ & 22 \\
+ GTS 100 & $17.7 \pm 0.4^{*}$ & 23 \\
+ GTS 300 & $16.0 \pm 0.2^{*}$ & 22 \\
+ Chol 5 & $17.2 \pm 0.2^{*}$ & 22 \\
+ Chol + GTS 300 & $15.8 \pm 0.3^{*}, \#$ & 24 \\
+ Chol 50 & $16.5 \pm 0.5^{*}$ & 22 \\
\hline
\end{tabular}

$* p<0.01, * * p<0.001$, significantly different from cholesterol-deprived group. ${ } p<0.01,{ }^{\#} p<0.001$, significantly different from cholesterol-fed group.

maximum survival days of worms grown in various treatments were summarized in Table 3. As shown in Fig. 3 and Table 3, worms grown in cholesterol-deprived medium showed a significantly shorter life span than those grown in cholesterol-fed medium, consistent with previous report. ${ }^{4,15)}$ Through life span study, we could observe that although worms grown in GTS supplement $(5,100$, or $300 \mu \mathrm{g} / \mathrm{ml})$ to cholesterol-deprived medium did not show a longer life span than those of cholesterol-fed group in F1, F2 and F3 generations, GTS supplement to cholesterol-deprived group produced significantly a longer life span of worms by about 3 $4 \mathrm{~d}$ than those of cholesterol-deprived group throughout F1 to F3 generations. The maximum survival day in GTS $(5,100$ or $300 \mu \mathrm{g} / \mathrm{ml})+$ cholesterol-deprived group was also extended by 3 to $9 \mathrm{~d}$ in $\mathrm{F} 1, \mathrm{~F} 2$, and F3 generations, respectively, compared with cholesterol-deprived group. Thus, throughout F1, F2 and F3 generations, GTS supplement in cholesteroldeprived medium restored normal life span as much as cholesterol-fed condition. However, supplement of GTS (300 $\mu \mathrm{g} / \mathrm{ml})$ to cholesterol-fed medium or supplement of ten-fold higher concentrations $(50 \mu \mathrm{g} / \mathrm{ml})$ of cholesterol to normal cholesterol $(5 \mu \mathrm{g} / \mathrm{ml})$-fed medium did not prolong the life span compared with cholesterol-fed group (Fig. 4, Table 3). Interestingly, we could observe that although GTS $(5 \mu \mathrm{g} / \mathrm{ml})$ 
A. F1

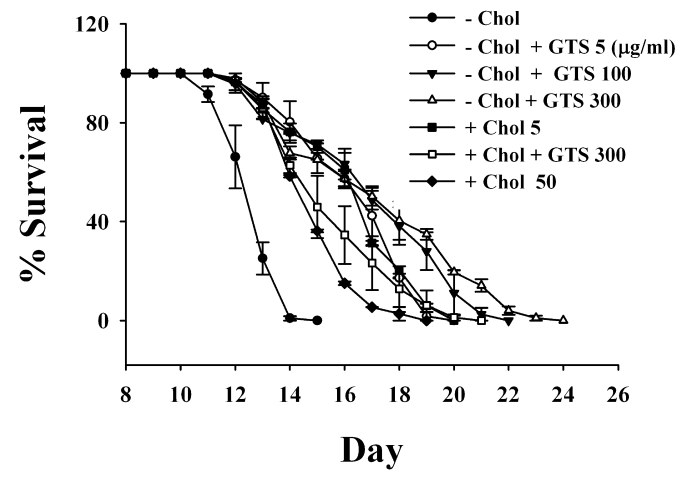

B. F2

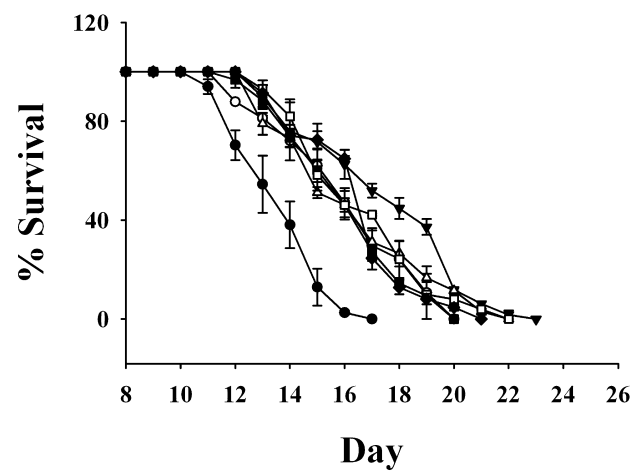

C. F3

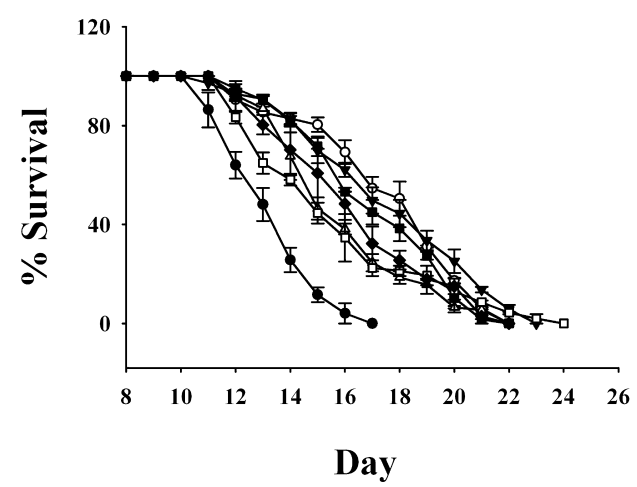

Fig. 3. Effects of GTS on Life Span of Worms Grown in CholesterolDeprived or -Fed Medium from F1 (A), F2 (B), and F3 (C) Generations at $20^{\circ} \mathrm{C}$

Life span of worms grown in indicated conditions was measured as described in Materials and Methods. Eggs laid by P0, F1, or F2 animals were placed on plates containing the Following treatments: - Chol; - Chol (cholesterol-fed, $50 \mu \mathrm{g} / \mathrm{ml})+\mathrm{GTS}(5 \mu \mathrm{g} /$ $\mathrm{ml}) ; \quad-$ Chol+GTS $(100 \mu \mathrm{g} / \mathrm{ml}) ;-$ Chol+GTS $(300 \mu \mathrm{g} / \mathrm{ml}) ; \quad+$ Chol; +Chol+GTS $(300 \mu \mathrm{g} / \mathrm{ml})$; +Chol (cholesterol-fed, $50 \mu \mathrm{g} / \mathrm{ml})$. The average values were obtained from $94-118$ worms

supplement in cholesterol-deprived medium did not restore reproduction, development, worm size, and growth rate as much as cholesterol-fed group, this concentration of GTS was enough for restoration of life span of worms grown in cholesterol-deprived medium. We could also observe that co-supplement of GTS to cholesterol-fed medium did not affect the life span of worms compared with worms grown in cholesterol-fed medium. Again, these results demonstrated that cholesterol deprivation in culture medium caused a short life span as shown in previous reports, ${ }^{4}$ ) but GTS supplement to cholesterol-deprived medium restored the normal life span.

Ginsenoside $\mathbf{R b}_{1}$ and $\mathbf{R c}$ Are Main Ingredients for the
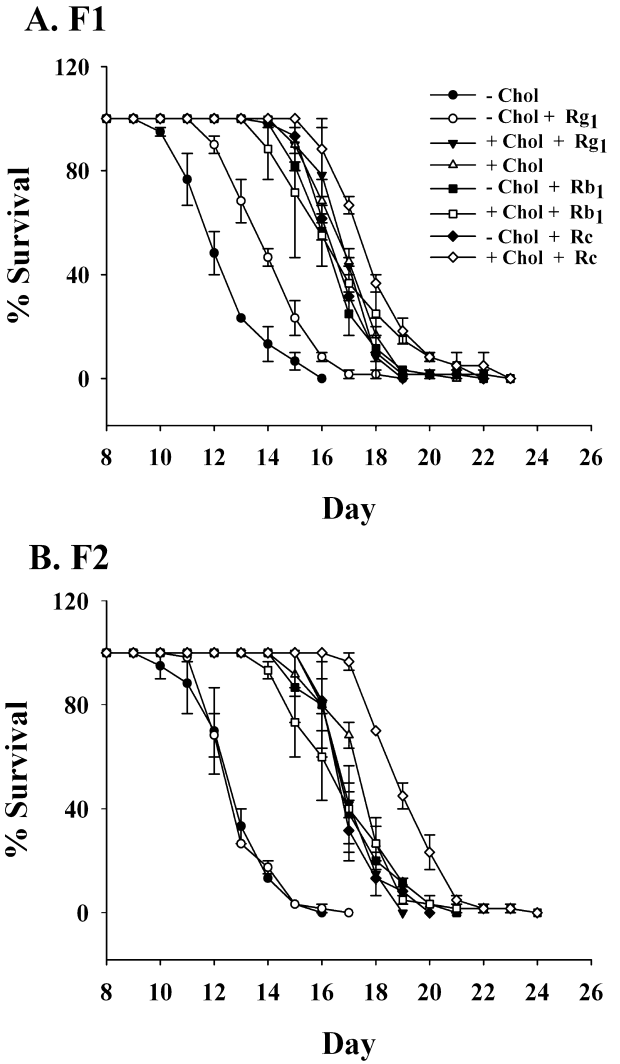

C. F3

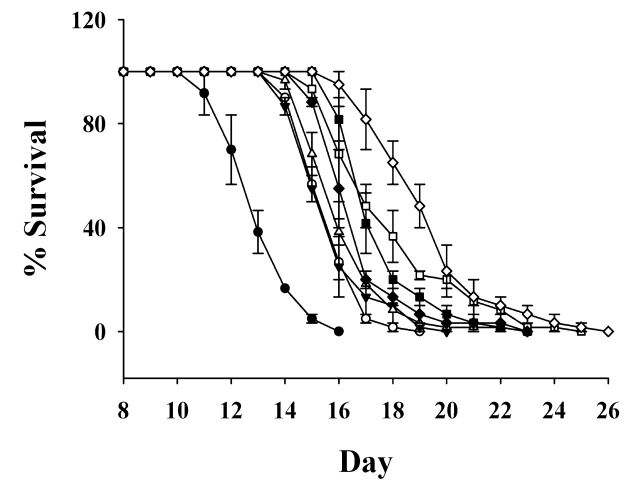

Fig. 4. Effects of Ginsenoside $\mathrm{Rb}_{1}$, Rc, or $\mathrm{Rg}_{1}$ on Life Span of Worms Grown in Cholesterol-Deprived or -Fed Medium from F1 (A), F2 (B), and F3 (C) Generations at $20^{\circ} \mathrm{C}$

Life span of worms grown in indicated conditions was measured as described in Materials and Methods and Fig. 3. We used ginsenoside $\mathrm{Rb}_{1}, \mathrm{Rc}$, or $\operatorname{Rg}_{1}(300 \mu \mathrm{M}$ each) The average values were obtained from sixty worms. The average values were obtained from $60-66$ worms.

Restoration of Life Span of Worms Grown in CholesterolDeprived Mediums and Ginsenoside Rc Prolonged the Life Span of Worms Grown in Normal Cholesterol-Fed Mediums Above results suggest that there might be active ginsenoside(s) responsible for life span restoration of worms grown in cholesterol-deprived medium in GTS. To identify active ingredient(s) for life span prolongation of worms, we tested the effects of several individual ginsenosides such as ginsenoside $\mathrm{Rb}_{1}, \mathrm{Rc}, \mathrm{Rg}_{1}, \mathrm{Rg}_{3}, \mathrm{Rh}_{1}$, and $\mathrm{Rh}_{2}(300 \mu \mathrm{M}$ each $)$ on life span of worms in cholesterol-deprived or fed-medium. Among them ginsenoside $\mathrm{Rg}_{3}, \mathrm{Rh}_{1}$, and $\mathrm{Rh}_{2}$, which are minor ginsenosides and are present as a trace of amount compared with ginsenoside $\mathrm{Rb}_{1}$ and $\mathrm{Rc}$, did not affect on life 
Table 4. Effects of Individual Ginsenosides on Life Span of C. elegans Grown in Cholesterol-Deprived and -Fed Medium

\begin{tabular}{|c|c|c|}
\hline \multirow{2}{*}{ F1 } & \multicolumn{2}{|c|}{ Life span } \\
\hline & Mean days & Maximum days \\
\hline- Chol & $12.6 \pm 0.1^{\# \#}$ & 16 \\
\hline- Chol+ $+\operatorname{Rg}_{1} 5(\mu \mathrm{M})$ & $12.6 \pm 0.1^{\#}$ & 17 \\
\hline$+\operatorname{Rg}_{1} 300$ & $14.4 \pm 0.2^{* \text {,\# }}$ & 19 \\
\hline$-\mathrm{Chol}+\mathrm{Rb}_{1} 5$ & $13.4 \pm 0.5$ & 19 \\
\hline$+\mathrm{Rb}_{1} 300$ & $16.8 \pm 0.6^{* *}$ & 23 \\
\hline- Chol+Rc 5 & $13.8 \pm 0.3$ & 19 \\
\hline$+\operatorname{Rc} 300$ & $17.0 \pm 0.1 * *$ & 22 \\
\hline+ Chol & $17.2 \pm 0.1 * *$ & 21 \\
\hline$+\mathrm{Chol}+\mathrm{Rg}_{1} 300$ & $17.2 \pm 0.3 * *$ & 19 \\
\hline$+\mathrm{Chol}+\mathrm{Rb}_{1} 300$ & $17.1 \pm 0.6^{*}$ & 22 \\
\hline$+\mathrm{Chol}+\mathrm{Rc} 300$ & $18.3 \pm 0.4 * *$ & 23 \\
\hline
\end{tabular}

\begin{tabular}{ccc}
\hline \hline \multirow{2}{*}{ F2 } & \multicolumn{2}{c}{ Life span } \\
\cline { 2 - 3 } & Mean days & Maximum days \\
\hline- Chol & $13.0 \pm 0.4^{\#}$ & 16 \\
- Chol $+\operatorname{Rg}_{1} 300$ & $13.2 \pm 0.1^{\# \#}$ & 17 \\
$+\operatorname{Rb}_{1} 300$ & $17.4 \pm 0.5^{*}$ & 21 \\
$+\operatorname{Rc} 300$ & $17.4 \pm 0.4^{*}$ & 20 \\
+ Chol & $17.8 \pm 0.4^{*}$ & 21 \\
+ Chol $+\operatorname{Rg}_{1} 300$ & $17.4 \pm 0.1^{* *}$ & 19 \\
+ Chol $+\operatorname{Rb}_{1} 300$ & $17.1 \pm 0.6^{*}$ & 24 \\
+ Chol $+\operatorname{Rc} 300$ & $19.4 \pm 0.1^{* *}$ & 24 \\
\hline
\end{tabular}

\begin{tabular}{|c|c|c|}
\hline \multirow{2}{*}{ F3 } & \multicolumn{2}{|c|}{ Life span } \\
\hline & Mean days & Maximum days \\
\hline -Chol & $13.2 \pm 0.3^{\#}$ & 16 \\
\hline$-\mathrm{Chol}+\mathrm{Rg}_{1} 300$ & $15.8 \pm 0.1^{*}$ & 19 \\
\hline$+\mathrm{Rb}_{1} 300$ & $17.7 \pm 0.3 * *$ & 23 \\
\hline$+\operatorname{Rc} 300$ & $16.9 \pm 0.4 *$ & 23 \\
\hline+ Chol & $16.4 \pm 0.3^{*}$ & 23 \\
\hline+ Chol $+\operatorname{Rg}_{1} 300$ & $15.9 \pm 0.3 *$ & 20 \\
\hline$+\mathrm{Chol}+\mathrm{Rb}_{1} 300$ & $18.1 \pm 0.4 * *$ & 25 \\
\hline+ Chol+Rc 300 & $19.5 \pm 0.7^{*, \#}$ & 26 \\
\hline
\end{tabular}

$* p<0.01, * * p<0.001$, significantly different from cholesterol-deprived group ${ }^{*} p<0.01, \# p<0.001$, significantly different from cholesterol-fed group.

span of worms grown in both cholesterol-deprived and cholesterol-fed medium rather inhibited the development and growth of worms (data not shown), whereas supplement of ginsenosides such as $\mathrm{Rb}_{1}$ or $\mathrm{Rc}$ but not $\mathrm{Rg}_{1}$ to cholesterol-deprived medium restored the life span as much as cholesterolfed group. The average life span and maximum days in F1, $\mathrm{F} 2$, and F3 generations of worms grown in cholesterol-deprived, cholesterol-fed, ginsenoside $\mathrm{Rb}_{1}, \mathrm{Rc}$, or $\mathrm{Rg}_{1}+$ cholesterol-deprived or -fed medium was summarized in Table 4. Interestingly, co-supplement of ginsenoside $\mathrm{Rc}$ but not $\mathrm{Rb}_{1}$ and $\mathrm{Rg}_{1}$ to cholesterol-fed medium not only prolonged the average life span but also extended the maximum days of life span by 2, 3 and $3 \mathrm{~d}$ compared with cholesterol-fed group in F1, F2, and F3 generations, respectively (Fig. 4, Table 4). Since low concentrations of GTS supplement $(5 \mu \mathrm{g} / \mathrm{ml})$ to cholesterol-deprived medium can substitute cholesterol for life span elongation (Table 3), we also tested the effects of ginsenoside $\mathrm{Rb}_{1}, \mathrm{Rc}$, or $\mathrm{Rg}_{1}$ at low concentration ( $5 \mu \mathrm{M}$ each) on worm life span in cholesterol-deprived medium. As

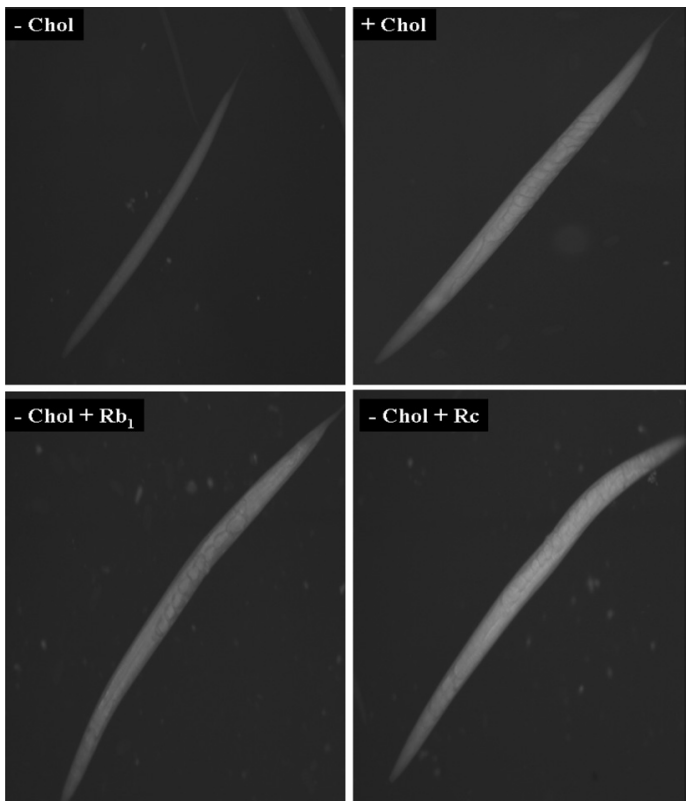

Fig. 5. Effects of Ginsenoside $\mathrm{Rb}_{1}$ or Rc on Filipin Staining of Worms Grown in Cholesterol-Deprived Medium

Eggs laid by F1 animals were placed on plates containing the following treatments: - Chol; +Chol; - Chol $+\mathrm{Rb}_{1}(300 \mu \mathrm{M}) ;-\mathrm{Chol}+\mathrm{Rc}(300 \mu \mathrm{M}$ supplement). Photographs were taken after growth for $72 \mathrm{~h}$ of F2 at $20^{\circ} \mathrm{C}$. All fluorescence micrographs of filipin staining of worms were taken at the same time under various treatment with $100 \times$ magnification.

shown in Table 4, we found that supplement of ginsenoside $\mathrm{Rb}_{1}, \mathrm{Rc}$, or $\mathrm{Rg}_{1}$ to cholesterol-deprived medium slightly but not significantly extended the life span of worm. Thus, we found among several individual ginsenosides tested that ginsenoside $R b_{1}$ and $R c$ at 300 but not $5 \mu \mathrm{M}$ are main ingredients for the restoration of life span of $C$. elegans grown in cholesterol-deprived mediums. We further found that ginsenoside $\mathrm{Rc}$ is the main component of Panax ginseng that prolongs the life span of $C$. elegans.

Effects of Ginsenoside $R b_{1}$ and Rc on Filipin Staining of $C$. elegans Grown in Cholesterol-Deprived Medium Above results show a possibility that ginsenoside $R b_{1}$ and $R c$ could be utilized as sterol substitute or sterol source by worms grown in cholesterol-deprived medium. If ginsenoside $\mathrm{Rb}_{1}$ and $\mathrm{Rc}$ are used as sterol source by $C$. elegans, there should be a difference in sterol staining when sterol in body of $C$. elegans is visualized. For this, we used filipin staining method. Filipin is a fluorescent polyene antibiotic that forms tight and specific complexes with $3 \beta$-hydroxy sterols. ${ }^{15-18)}$ As shown in Fig. 5, we could observe that worms grown in ginsenoside $\mathrm{Rb}_{1}$ or $\mathrm{Rc}(300 \mu \mathrm{M})+$ cholesterol-deprived medium were much bigger than those grown in cholesterol-deprived medium and that exhibited strong filipin staining of whole body of worms with discrete internal organs compared with those grown in cholesterol-deprived medium. These results show a possibility that worms might at least modify ginsenoside $\mathrm{Rb}_{1}$ and $\mathrm{Rc}$ into $3 \beta$-hydroxy sterols that could react with filipin, although ginsenoside $\mathrm{Rb}_{1}$ and $\mathrm{Rc}$ have no $3 \beta$-hydroxyl group (Fig. 1). In addition, since co-supplement of GTS to cholesterol-fed medium produces bigger worms in size and diameter than those grown in cholesterol-fed medium (Fig. 2), we tested whether co-supplement of ginsenoside $\mathrm{Rb}_{1}$ or $\mathrm{Rc}(300 \mu \mathrm{M})$ to cholesterol-fed medium also 
produces the same effects. In contrast to GTS, we found that co-supplement of ginsenoside $\mathrm{Rb}_{1}$ or $\mathrm{Rc}$ to cholesterol-fed medium did not affect worm sizes compared with those grown in cholesterol-fed medium (data not shown). These results show a possibility that the productions of bigger worms in GTS co-supplement to cholesterol-fed medium might be achieved through total effects of individual ginsenosides rather than those of individual ginsenosides.

\section{DISCUSSION}

Nematodes, including free-living C. elegans, require sterol for its normal development, growths, and life span as a nutritional source, ${ }^{19)}$ since $C$. elegans is unable to biosynthesize sterol de novo. ${ }^{19)}$ However, C. elegans is usually able to obtain cholesterol or cholesterol-like sterols for their growth by metabolizing natural sterols such as phytosterols present in many plants or sterols from the animal body in the soil. ${ }^{20)}$ Although some species of nematodes are known to be parasitic in wild and cultivated ginseng roots, ${ }^{5)}$ little is known on the physiological roles of ginseng saponins or ginsenosides to the reproduction, development, growth, and life span of $C$. elegans.

In the present study, we investigated the effect of GTS on the average brood size, growth rate, percent development, and life span of $C$. elegans in cholesterol-deprived or -fed medium. Our results revealed five major findings. First, we found that cholesterol deprivation caused a significant reduction of average brood size, whereas GTS supplement to cholesterol-deprived medium restored the average brood size of C. elegans as much as cholesterol-fed medium. Moreover, we found that co-supplement of GTS with cholesterol significantly increased the average brood size (Table 1). These results further indicate that GTS directly affects egg laying ability of $C$. elegans. Second, cholesterol deprivation caused severe damages on percent development, whereas GTS supplement to cholesterol-deprived medium also restored worm development to adulthood. Third, cholesterol deprivation caused a significant reduction in worm size and retarded growth compared with cholesterol-fed group, whereas GTS supplement to cholesterol-deprived group restored worm size and growth as much as cholesterol-fed group throughout F1 to F3 generations. Moreover, co-supplement of GTS with cholesterol produced bigger worms and promoted rapid growth than only cholesterol-fed group (Fig. 2, Table 2). Fourth, life span of worms grown in cholesterol deprivation medium was significantly shorter than those of cholesterolfed group. However, even low amount of GTS supplement $(5 \mu \mathrm{g} / \mathrm{ml})$ to cholesterol-deprived group extended life span as much as cholesterol-fed group (Table 3). Fifth, we found that ginsenoside $R b_{1}$ and $R c$ were main ingredients that are responsible for GTS beneficial effects to worms grown in cholesterol-deprived medium. Interestingly, co-supplement of ginsenoside Rc further extended life span and maximal survival days of worms grown in cholesterol-fed medium. However, we could not observe the same life span extending effects in worms grown in medium supplemented with tenfold higher concentration $(50 \mu \mathrm{g} / \mathrm{ml})$ of cholesterol than normal medium (Fig. 3, Table 3). These results indicate that $C$. elegans could use GTS and its individual ginsenosides as a sterol substitute for its normal fertility, development and growth. Further ginsenoside Rc has life span extending effect in C. elegans.

Although there are many publications on the presence of saponins from various kinds of plants, the physiological roles of saponins in plants are not yet fully understood. ${ }^{21)}$ Recent studies showed that most of saponins are usually known to be antimicrobial, to inhibit mould, and to protect plants from insect attack. For example, it is known that saponins such as avenacosides in oat are activated by the plant's enzymes in response to tissue damages or pathogen attacks. ${ }^{22}$ ) Thus, most of saponins may be considered a part of plants' defense systems and have been included in a large group of protective molecules found in plants named 'phytoanticipins' or 'phytoprotectants'. ${ }^{23)}$

However, we found in the present study that GTS helps $C$. elegans survive normally rather than does harm to them, since GTS supplement supports worm egg laying, growth, and development and extended life span in spite of cholesterol deprivation. One speculation on these beneficial effects of GTS might be due to the chemical properties of GTS. Recent accumulating evidences showed that ginseng saponins in worms mimic the actions of steroid hormones such as estrogen and progesterone as in mammalian cells. ${ }^{24)}$ Thus, these steroidal actions of ginseng saponins might directly meet sterol requirement as sterol source. The other speculation is that $C$. elegans might modify ginseng saponins present in medium in sterol-deprivation environments and produces sterols from ginseng saponins for its utilization. This speculation might be supported from filipin staining. We used sterol-specific filipin staining, which shows fluorescence upon forming a complex with 3-hydroxysterols, ${ }^{12)}$ to detect sterol accumulation in C. elegans. ${ }^{16-18,25)}$ As shown Fig. 5, worms grown in ginsenoside $\mathrm{Rb}_{1}$ or $\mathrm{Rc}$ supplement were bigger than those grown in cholesterol-deprived medium and exhibited stronger fluorescence in whole body with discrete internal organs than those grown in cholesteroldeprived medium, although ginsenoside $\mathrm{Rb}_{1}$ and $\mathrm{Rc}$ have no 3-hydroxy group at carbon-3 instead have two carbohydrates (Fig. 1). Moreover, treatment of azacoprostane- $\mathrm{HCl}$, which is known as $\Delta$-dehydrocholesterol reductase inhibitor, ${ }^{26}$ blocked the beneficial effects (i.e. normal fertility, development, growth, and life span) of GTS to cholesterol-deprived group (data not shown). These results suggest that $C$. elegans might have metabolic pathways for modification of GTS, ginsenoside $\mathrm{Rb}_{1}$, or $\mathrm{Rc}$ in order to produce its required sterol. However, we could not exclude other possibilities that GTS or ginsenosides might participate in other physiological roles besides sterol substitute, since co-supplement of GTS to cholesterol-fed medium produces much larger brood size and bigger worms than those observed in cholesterol-fed medium (Fig. 2). Moreover, low concentration of GTS supplement to cholesterol-deprived group significantly extended life span compared with cholesterol-deprived group (Fig. 3, Table 3), although low concentration of individual ginsenosides such as $\mathrm{Rb}_{1}, \mathrm{Rc}$ or $\mathrm{Rg}_{1}$ had no significant effects (Table 4). However, co-supplement of ginsenoside Rc $(300 \mu \mathrm{M})$ to cholesterol-fed medium also induced prolongation of life span (Fig. 4), which was not observed in much higher concentration of cholesterol, indicating that ginsenoside Rc has life span extending effect in C. elegans (Table 4). Future studies will be further required to elucidate the physiological roles and 
metabolic pathway of GTS or ginsenosides in C. elegans.

Among individual ginsenoside $\mathrm{Rb}_{1}, \mathrm{Rc}$ and $\mathrm{Rg}_{1}$, we could observe that life span extending effect of ginsenoside $\mathrm{Rg}_{1}$ on worms grown in cholesterol-deprived or fed-medium was less effective than those of ginsenoside $\mathrm{Rb}_{1}$ and Rc (Fig. 4). As shown in Fig. 1, the differences of chemical structure between ginsenoside $\mathrm{Rg}_{1}$ and $\mathrm{Rb}_{1}$ or $\mathrm{Rc}$ are that ginsenoside $\mathrm{Rg}_{1}$ has one carbohydrate at carbon- 6 and has hydroxyl group at carbon-3, whereas ginsenoside $\mathrm{Rb}_{1}$ and $\mathrm{Rc}$ has only two carbohydrates at carbon-3. In addition to life span, we could also observe that ginsenoside $\mathrm{Rb}_{1}$ or $\mathrm{Rc}$ but not $\mathrm{Rg}_{1}$ was effective to restore the average brood size, development, growth rate, and worm size of worms grown in cholesteroldeprived medium as GTS did (data not shown). Thus, the carbohydrate attached at carbon- 6 of ginsenoside $\mathrm{Rg}_{1}$ might play a role in exhibiting less physiologically beneficial effects in prolonging the life span, reproduction, and development of worms grown in cholesterol-deprived or fedmedium.

In summary, using $C$. elegans as model system we herein used GTS and individual ginsenosides to know its role in cholesterol-deprived or -fed medium. We have obtained novel evidences for the first time that GTS could substitute cholesterol in reproduction, growth, and development of $C$. elegans and that supplement of GTS, ginsenoside $\mathrm{Rb}_{1}$ or Rc to cholesterol-deprived medium extended life span as much as worms grown in cholesterol-fed medium. We also found that supplement of ginsenoside Rc to cholesterol-fed group further extended life span in cholesterol-fed group. These novel findings provide new insights that $C$. elegans utilizes subtypes of ginsenosides as sterol source.

Acknowledgements This study was supported by grants of 2007 Korea Ginseng Corporation, BK21 Project of the Korea Research Foundation, the Neurobiology Research Program from the Korea Science and Engineering Foundation (KOSEF), the Bio/Molecular Informatics Center of Konkuk University (KRF-2006-005-J03403), Technology Development Program for Agriculture and Forestry, Ministry of Agriculture and Forestry, Republic of Korea, and On-Site Cooperative Agriculture Research Project (Project No.
20070101080014) to J. Ahn, RDA, Korea.

\section{REFERENCES}

1) Tyler V. E., J. Pharm. Technol., 11, 214-220 (1995).

2) Hieb W. F., Rothstein M., Science, 160, 778-880 (1968).

3) Merris M., Wadsworth W. G., Khamrai U., Bittman R., Chitwood D. J., Lenard J., J. Lipid Res., 44, 172-181 (2003).

4) Shim Y. H., Chun J. H., Lee E. Y., Paik Y. K., Mol. Reprod. Dev., 61, $358-366$ (2002).

5) Kim J. H., Jeon Y. H., Park H., Lee B. D., Choi D. H., Park B. Y., Khan Z., Kim Y. H., Nematology, 8, 637-639 (2006).

6) Tanaka O., Nagai M., Shibata S., Chem. Pharm. Bull., 14, 1157-1161 (1966).

7) Shibata S., Tanaka O., Ando T., Sado M., Tsushima S., Osawa T., Chem. Pharm. Bull., 14, 1150-1156 (1966).

8) Kim S. K., Kwak Y. S., Kim S. W., Hwang S. Y., Ko Y. S., Yoo C. M., J. Ginseng Res., 22, 155-160 (1998).

9) Shibata S., Proceedings of International Ginseng Symposium, 1974, pp. $69-76$.

10) Brenner S., Genetics, 77, 71-94 (1974).

11) Matyash V., Entchev E. V., Mende F., Wilsch-Brauninger M., Thiele C., Schmidt A. W., Knolker H. J., Ward S., Kurzchalia T. V., PLoS. Biol., 2, 1561-1571 (2004).

12) Merris M., Wadsworth W. G., Khamrai U., Bittman R., Chitwood D. J., Lenard J., J. Lipid Res., 44, 172-181 (2002).

13) Beanan M. J., Strome S., Development, 116, 755-766 (1992).

14) Miller D. M., Shakes D. C., Methods Cell Biol., 48, 365-394 (1995).

15) Merris M., Kraeft J., Tint G. S., Lenard J., J. Lipid Res., 45, 2044 2051 (2004).

16) Bittman R., Lipids, 13, 686-691 (1978).

17) Norman A. W., Spielvogel A. M., Wong R. G., Adv. Lipid Res., 14, 127-170 (1976)

18) Pelletier R. M., Vitale M. L., J. Histochem. Cytochem., 42, 15391554 (1994).

19) Rothstein M., Comp. Biochem. Physiol., 27, 309-317 (1968).

20) Chitwood D. J., Lusby W. R., Thompson M. J., Kochansky J. P., Howarth O. W., Lipids, 30, 567-573 (1995).

21) Francis G., Kerem Z., Makkar H. P. S., Becker K., Br. J. Nutr., 88, 587-605 (2002).

22) Gus-Mayer S., Brunner H., Schneider-Poetsch H. A., Rudiger W., Plant Mol. Biol., 26, 909-921 (1994).

23) Morrissey J. P., Osbourn A. E., Mol. Biol. Rev., 63, 708 -724 (1999).

24) Cho J., Park W., Lee S., Ahn W., Lee Y., Clin. Endocrinol. Metab., 89, 3510 -3515 (2004).

25) Severs N. J., Subcell Biochem., 28, 477-505 (1997).

26) Choi B. K., Chitwood D. J., Paik Y. K., Mol. Cell. Proteomics, 2, 1086-1095 (2003). 